\title{
COMMENTARY
}

\section{Immunity, inflammation and sepsis: new insights and persistent questions}

\author{
W Joshua Frazier* \\ See related research by Giamarellos-Bourboulis et al., http://ccforum.com/content/15/1/R27
}

\begin{abstract}
Sepsis is now understood to affect a variety of changes in the host, chief among them being alterations in immune system function. Proper immune function involves a competent proinflammatory response to stimuli as well as a regulated counteracting force to restore homeostasis and prevent systemic inflammation and organ dysfunction. Broad-spectrum suppression of the inflammatory response has not been shown to be beneficial for patients suffering from septic disease. In fact, sepsis-related immune suppression has become increasingly recognized as an important contributor to late morbidity and mortality in the critically ill. Giamarellos-Bourboulis and colleagues detail the impaired ability of septic patients to produce proinflammatory cytokines upon ex vivo stimulation, and introduce altered caspase-1 activity as potentially contributory to this process. Proper understanding of the cellular and molecular events resulting in immune suppression following sepsis is important in the identification of new strategies for treatment and the ideal timing of therapy.
\end{abstract}

In their most recent work, Giamarellos-Bourboulis and colleagues examine sepsis-related immune dysfunction by assessing the proinflammatory cytokine production capacity in septic patients and healthy volunteers following ex vivo stimulation [1]. A critical examination of their article not only reveals new insights into the possible mechanisms underlying sepsis-related immune system dysfunction, but also highlights the difficulties inherent in this line of inquiry. The authors report that septic

*Correspondence: warren.frazier@nationwidechildrens.org

The Ohio State University College of Medicine, Division of Critical Care Medicine, Nationwide Children's Hospital, Center for Perinatal Research, The Research Institute at Nationwide Children's Hospital, 700 Children's Drive, Columbus, $\mathrm{OH}$ 43205, USA patients' isolated leukocytes produced lower amounts of the proinflammatory cytokines IL- $1 \beta$, IL- 6 and, to a lesser extent, TNF $\alpha$ when stimulated within 24 hours of Gram-negative sepsis onset. Interestingly, greater severity of sepsis was correlated with a greater degree of impairment. Results were similar in healthy volunteers challenged with lipopolysaccharide, indicating an early shift to an immune-suppressed phenotype following systemic inflammation.

In our current understanding, an early anti-inflammatory phenotype following close on the heels of a severe inflammatory challenge (sepsis, trauma, burn injury, and so forth) acts as a counterweight to the systemic inflammatory response syndrome and restores immune homeostasis [2]. When short-lived and self-limited, this anti-inflammatory surge is best termed the compensatory anti-inflammatory response syndrome (CARS). In some patients, however, CARS is pathologically exaggerated and prolonged (beyond 48 hours). This persistent state known as immunoparalysis - is associated with increased rates of nosocomial sepsis, multiorgan dysfunction syndrome and death in critically ill patients [3-5]. Although the features are similar, the distinction between CARS and immunoparalysis is important when contemplating therapy. The issue is one of timing: to reverse physiologic CARS too early in the course of illness risks further inflammatory insult. The literature, however, suggests that pathologic immunoparalysis is reversible, to the benefit of the patient, without iatrogenic hyperinflammation [6-8].

Although the identification of immunoparalysis, its impact on outcome, and its potential reversibility are becoming increasingly understood, relatively little is known about the cellular and molecular mechanisms driving the process. Previous authors have investigated the mechanisms of immunoparalysis at the transcriptome level [9-11]. This current report makes the case for posttranslational forces as well. In addition to ex vivo cytokine production, Giamarellos-Bourboulis and colleagues examined levels of caspase- 1 , a protease that cleaves proIL-1 $\beta$ into its active form. In their septic patients, reduced IL-1 $\beta$ production capacity was associated with 
markedly diminished caspase-1 levels. In their healthy volunteers, injection with lipopolysaccharide resulted in lower levels of caspase-1, as well as impaired production of IL-1 $\beta$. Heretofore, immunoparalysis research has largely centered on the canonical proinflammatory cytokine TNF $\alpha$. (Indeed, assessing ex vivo lipopolysaccharide-induced TNF $\alpha$ production capacity by isolated monocytes is a commonly used method for diagnosing immunoparalysis [2].) By introducing altered IL-1 $\beta$ biology as a contributory process in the development of CARS/immunoparalysis, the authors lay the ground work for additional avenues of research into the cause(s) or diagnosis of these conditions. Further research is necessary to confirm a causal link between impaired caspase- 1 function and IL- $1 \beta$ production. It is also important to note that, like TNF $\alpha$, IL-1 $\beta$ is one of myriad proinflammatory cytokines whose production and regulation may be altered in immunoparalysis.

Despite these new and interesting contributions, placing the current work by Giamarellos-Bourboulis and colleagues into proper context is complicated by their study design. The early (within 24 hours) sampling window, without additional longitudinal samples, makes it impossible to distinguish patients with physiologic CARS from those with pathologic immunoparalysis. Previous evidence has shown that only patients unable to reverse an early anti-inflammatory phenotype by 3 to 4 days are at increased risk of poor outcome [3]. An early anti-inflammatory phenotype (possible CARS) is thus apparently not as concerning as prolonged immune suppression (probably immunoparalysis). Unfortunately, the mechanisms or predispositions mediating the transition from CARS to immunoparalysis are currently unknown.

The work by Giamarellos-Bourboulis and colleagues, and that of many other researchers, seeks to uncover the events surrounding sepsis-related immune system dysfunction. By understanding the mechanisms involved, such as altered caspase-1 action, novel therapeutic targets may be identified. Through careful targeting of therapy to those patients with true immunoparalysis, the best chance for improved outcomes will be realized.

Abbreviations

CARS, compensatory anti-inflammatory response syndrome; IL, interleukin; TNF, tumor necrosis factor.
Competing interests

The author declares that he has no competing interests.

\section{Acknowledgements}

WJF receives funding from the Child Health Research Career Development Award K12 HD043372-09, and from the Research Institute at Nationwide Children's Hospital.

Published: 24 February 2011

\section{References}

1. Giamarellos-Bourboulis EJ, van de Veerdonk FL, Mouktaroudi M, Raftogiannis M, Antonopoulou A, Joosten LA, Pickkers P, Savva A, Georgitsi M, van der Meer JW, Netea MG: Inhibition of caspase-1 activation in Gram-negative sepsis and experimental endotoxemia. Crit Care 2011, 15:R27.

2. Frazier WJ, Hall MW: Immunoparalysis and adverse outcomes from critical illness. Pediatr Clin North Am 2008, 55:647-668, xi.

3. Monneret G, Lepape A, Voirin N, Bohe J, Venet F, Debard AL, Thizy H, Bienvenu J, Gueyffier F, Vanhems P: Persisting low monocyte human leukocyte antigen-DR expression predicts mortality in septic shock. Intensive Care Med 2006, 32:1175-1183.

4. Polk HC, Jr, Wellhausen SR, Regan M: A systematic study of host defense processes in badly injured patients. Ann Surg 1986, 204:282-297.

5. Volk HD, Reinke P, Krausch D, Zuckermann H, Asadullah K, Muller JM, Docke WD, Kox WJ: Monocyte deactivation - rationale for a new therapeutic strategy in sepsis. Intensive Care Med 1996, 22(Suppl 4):S474-S481.

6. Nakos G, Malamou-Mitsi VD, Lachana A, Karassavoglou A, Kitsiouli E, Agnandi $\mathrm{N}$, Lekka ME: Immunoparalysis in patients with severe trauma and the effect of inhaled interferon-gamma. Crit Care Med 2002, 30:1488-1494.

7. Nierhaus A, Montag B, Timmler N, Frings DP, Gutensohn K, Jung R, Schneider CG, Pothmann W, Brassel AK, Schulte Am Esch J: Reversal of immunoparalysis by recombinant human granulocyte-macrophage colony-stimulating factor in patients with severe sepsis. Intensive Care Med 2003, 29:646-651.

8. Rosenbloom AJ, Linden PK, Dorrance A, Penkosky N, Cohen-Melamed MH, Pinsky MR: Effect of granulocyte-monocyte colony-stimulating factor therapy on leukocyte function and clearance of serious infection in nonneutropenic patients. Chest 2005, 127:2139-2150.

9. Hall MW, Gavrilin MA, Knatz NL, Duncan MD, Fernandez SA, Wewers MD: Monocyte mRNA phenotype and adverse outcomes from pediatric multiple organ dysfunction syndrome. Pediatr Res 2007, 62:597-603.

10. Hall MW, Gavrilin MA, Knatz NL, Duncan MD, Wewers MD: Monocyte mRNA expression in pediatric multiple organ dysfunction syndrome: antiinflammatory dominance in nonsurvivors. Crit Care Med 2005, 33:A152

11. Pachot A, Lepape A, Vey S, Bienvenu J, Mougin B, Monneret G: Systemic transcriptional analysis in survivor and non-survivor septic shock patients: a preliminary study. Immunol Lett 2006, 106:63-71.

doi:10.1186/cc10028

Cite this article as: Frazier WJ: Immunity, inflammation and sepsis: new

insights and persistent questions. Critical Care 2011, 15:124. 